\title{
Relationship between tumor location, size, and WHO grade in meningioma
}

\author{
*Stephen T. Magill, MD, PhD, Jacob S. Young, MD, Ricky Chae, Manish K. Aghi, MD, PhD, \\ Philip V. Theodosopoulos, MD, and Michael W. McDermott, MD \\ Department of Neurological Surgery, University of California, San Francisco, California
}

\begin{abstract}
OBJECTIVE Prior studies have investigated preoperative risk factors for meningioma; however, no association has been shown between meningioma tumor size and tumor grade. The objective of this study was to investigate the relationship between tumor size and grade in a large single-center study of patients undergoing meningioma resection.

METHODS A retrospective chart review of patients undergoing meningioma resection at the University of California, San Francisco, between 1985 and 2015 was performed. Patients with incomplete information, spinal meningiomas, multiple meningiomas, or WHO grade III meningiomas were excluded. The largest tumor dimension was used as a surrogate for tumor size. Univariate and multivariate logistic regression models were used to investigate the relationship between tumor grade and tumor size. A recursive partitioning analysis was performed to identify groups at higher risk for atypical (WHO grade II) meningioma.

RESULTS Of the 1113 patients identified, $905(81 \%)$ had a WHO grade I tumor and in $208(19 \%)$ the tumors were WHO grade II. The median largest tumor dimension was $3.6 \mathrm{~cm}$ (range $0.2-13 \mathrm{~cm}$ ). Tumors were distributed as follows: skull base $(n=573,51 \%)$, convexity/falx/parasagittal $(n=431,39 \%)$, and other $(n=109,10 \%)$. On univariate regression, larger tumor size $(p<0.001)$, convexity/falx/parasagittal location $(p<0.001)$, and male sex $(p<0.001)$ were significant predictors of WHO grade II pathology. After controlling for interactions, multivariate regression found male sex (OR 1.74, $95 \% \mathrm{Cl} 1.25-2.43$ ), size 3-6 cm (OR 1.69, 95\% Cl 1.08-2.66), size > $6 \mathrm{~cm}$ (OR 3.01, 95\% Cl 1.53-5.94), and convexityl falx/parasagittal location (OR 1.83, 95\% Cl 1.19-2.82) to be significantly associated with WHO grade II. Recursive partitioning analysis identified male patients with tumors $>3 \mathrm{~cm}$ as a high-risk group (32\%) for WHO grade II meningioma.
\end{abstract}

CONCLUSIONS Larger tumor size is associated with a greater likelihood of a meningioma being WHO grade II, independent of tumor location and male sex, which are known risk factors.

https://thejns.org/doi/abs/10.3171/2018.1.FOCUS17752

KEY WORDS meningioma; convexity; parasagittal; skull base; tumor size; atypical; grade; location

$\mathrm{M}$ ENINGIOMAS are the most common primary intracranial neoplasm. They are more common in women and their incidence rises with increasing age. ${ }^{3,5,10}$ Meningiomas are categorized into 3 histopathological grades by the WHO, with 70\%-95\% of meningiomas being classified as WHO grade I. ${ }^{15,17,18,20}$ Symptomatic tumors are treated primarily with surgery or radiotherapy. The WHO grade II and III meningiomas are more aggressive, with higher rates of mitosis, and are more likely to recur after surgery and require adjuvant treatment with radiotherapy ${ }^{23}$ Therefore, determining preoperative risk factors for higher tumor grade can provide useful information for both neurosurgeons and patients.
Prior studies have found risk factors for high-grade meningiomas. Male sex and convexity location are known to be associated with increased likelihood of a meningioma being atypical. ${ }^{19}$ Although convexity meningiomas are more commonly grade II or III compared to skull base meningiomas, atypical tumors do occur along the skull base. Liang et al. reported that up to $19 \%$ of skull base meningiomas were either grade II or III. ${ }^{19}$ Radiographic findings, including brain invasion, bone invasion, and peritumoral edema in the surrounding brain, have also been associated with higher-grade meningiomas. ${ }^{22}$ However, to our knowledge no study has shown that size is independently associated with tumor grade. Interestingly, in our

ABBREVIATIONS RPA = recursive partitioning analysis.

SUBMITTED December 1, 2017. ACCEPTED January 8, 2018.

INCLUDE WHEN CITING DOI: 10.3171/2018.1.FOCUS17752.

* S.T.M. and J.S.Y. contributed equally to this study. 
experience we anecdotally observed that larger tumors tended to be higher grade. Thus, we performed a retrospective study to evaluate whether tumor size was associated with tumor grade.

\section{Methods}

\section{Study Design, Setting, Size, and Participants}

We performed a retrospective chart review of patients who underwent resection of meningioma at the University of California, San Francisco, between 1985 and 2016. Variables were collected from provider notes, pathology records, operative notes, and radiological reports. Patients were excluded if no size information was available, the tumor was located in the spine, or the tumor was WHO grade III. Grade III tumors were removed due to their rarity in our data set. The manuscript was drafted consistent with the STROBE (Strengthening the Reporting of Observational Studies in Epidemiology) statement. ${ }^{28}$ The institutional review board at our institution approved this study.

\section{Demographic and Tumor Variables}

Demographic variables included age, sex, and followup duration. Tumor grade was obtained from the pathology report at the time of resection, and thus tumors resected prior to 2007 will be biased toward WHO grade I due to the 2007 WHO grading changes. Tumor size was determined from the preoperative MRI scan and radiologist report. The largest diameter in either the anterior-posterior, transverse, or craniocaudal dimension was used as an overall surrogate for tumor size. Tumor locations were obtained from operative and radiology reports, which included convexity, falx, parasagittal, tentorium, cavernous sinus, clinoid, parasellar, tuberculum sellae, planum sphenoidale, olfactory groove, orbital, middle fossa, sphenoid wing, clivus, cerebellopontine angle, posterior fossa, petroclival, petrous, foramen magnum, jugular foramen, skull base, intraventricular, and multifocal. The locations were further categorized into 3 groups: convexity/falx/ parasagittal, skull base tumors, and other. Skull base location was defined as cavernous sinus, cerebellopontine angle, clinoid, clivus, foramen magnum, jugular foramen, middle fossa, olfactory groove, orbital, parasellar, petroclival, petrous, planum sphenoidale, posterior fossa, skull base, sphenoid wing, and tuberculum sellae. Other locations included intraventricular and multifocal tumors that could not be easily classified into skull base, convexity, or falx locations.

\section{Study Bias}

This is a retrospective study, and thus is limited by selection bias. No patients were excluded if they had information regarding the variables of interest in the chart, thus limiting selection bias.

\section{Statistical Analysis}

All statistical analyses were performed using JMP Pro (version 13.0; SAS Institute, Inc.). Demographic data were assembled and analyzed in the standard fashion. For cat- egorical data, Pearson's chi-square (multiple categories) or Fisher exact (2 categories) tests were reported. Univariate and multivariate logistic regression was performed and used to calculate odds ratios. A recursive partitioning analysis (RPA) was performed to split the continuous size data into clinically relevant groups. The RPA was also used with multiple variables to determine groups at risk for having a WHO grade II tumor. The number of partitions performed was based on maximal $\mathrm{R}^{2}$ as calculated by $\mathrm{k}$-fold cross-validation $(\mathrm{k}=5)$.

\section{Results}

\section{Demographics and Tumor Characteristics}

A final sample size of 1113 patients (mean age 55.7 years, range $8-90$ years, $73.9 \%$ female) was obtained from the electronic medical record (Table 1). The majority of patients presented with either a headache or cranial nerve deficit. The WHO grade at the time of resection was used to assign the WHO grade. The majority (81\%) of patients had a WHO grade I tumor and $19 \%$ were WHO grade II. The mean tumor size was $3.8 \mathrm{~cm}$ (SD $1.8 \mathrm{~cm}$ ), and the median size was $3.6 \mathrm{~cm}$ (range $0.2-13 \mathrm{~cm}$ ). Roughly half of the patients in our study had meningiomas located in the skull base, $40 \%$ of tumors were located in the falx/ parasagittal or convexity area, and the remaining $10 \%$ of tumors were found in other locations (Table 2).

\section{Risk Factors for WHO Grade II}

We performed univariate logistic regression to determine if age, sex, tumor location, and tumor size were significantly associated with WHO grade (Table 3 ). Age was not a significant predictor of grade $(p=0.61)$. Tumor size was significantly associated with a meningioma being WHO grade II (Fig. 1; p < 0.001). Tumor convexity/ falx/parasagittal location (OR 1.90, 95\% CI 1.38-2.63, p $<0.001$ ) and other location (OR 1.71, 95\% CI 1.02-2.86,

TABLE 1. Characteristics in 1113 patients with meningioma

\begin{tabular}{lc}
\hline \multicolumn{1}{c}{ Characteristic } & Value \\
\hline No. of patients & 1113 \\
\hline Median age in yrs (range) & $55.7(8-90)$ \\
\hline Female & $822(73.9 \%)$ \\
\hline Initial presenting symptoms & \\
\hline Headache & $408(36.7 \%)$ \\
\hline Focal cranial nerve deficit & $348(31.3 \%)$ \\
\hline Seizure & $188(16.9 \%)$ \\
\hline Cognitive change & $160(14.4 \%)$ \\
\hline Weakness & $123(11.1 \%)$ \\
\hline Vertigo/dizziness & $109(9.8 \%)$ \\
\hline Incidental & $80(7.2 \%)$ \\
\hline Ataxia/gait change & $70(6.3 \%)$ \\
\hline Pain/sensory change & $62(5.6 \%)$ \\
\hline Proptosis & $23(2.1 \%)$ \\
\hline Syncope & $11(1.0 \%)$ \\
\hline Not recorded & $160(14.4 \%)$ \\
\hline
\end{tabular}


TABLE 2. Tumor characteristics

\begin{tabular}{lccc}
\hline \multicolumn{1}{c}{ Tumor Location } & $\begin{array}{c}\text { No. of } \\
\text { Patients }\end{array}$ & $\begin{array}{c}\text { Median } \\
\text { Diameter }(\mathrm{cm})\end{array}$ & $\begin{array}{c}\text { WHO } \\
\text { Grade II }\end{array}$ \\
\hline Skull base & 573 & & \\
\hline$\quad$ Anterior cranial fossa & 152 & 2.7 & $9.9 \%$ \\
\hline$\quad$ Middle cranial fossa & 191 & 3.6 & $18.8 \%$ \\
\hline$\quad$ Posterior cranial fossa & 230 & 3.0 & $13.0 \%$ \\
\hline Convexity/falx/parasagittal & & & \\
\hline$\quad$ Falx/parasagittal & 212 & 4.5 & $20.3 \%$ \\
\hline$\quad$ Convexity & 219 & 3.7 & $27.4 \%$ \\
\hline$\quad$ Other & 109 & 3.8 & $22.0 \%$ \\
\hline Total & 1113 & 3.6 & $18.7 \%$ \\
\hline
\end{tabular}

$\mathrm{p}=0.03$ ) were also significantly associated with a meningioma being WHO grade II compared to skull base meningiomas. Finally, male sex was also associated with a meningioma being WHO grade II (OR 1.94, 95\% CI 1.41$2.69, \mathrm{p}<0.001)$. Next, we performed multivariate logistic analysis and included all significant variables from the univariate model. Given the relationship between tumor location and WHO grade II seen on univariate analysis, we crossed tumor size with location. Tumor size remained significantly associated with tumor grade, even with the crossed variables in the model: tumor size $(p=0.02$ for size between 3.0 and $6.0 \mathrm{~cm} ; \mathrm{p}=0.002$ for size $>6.0 \mathrm{~cm}$ compared to tumors $<3.0 \mathrm{~cm})$ and tumor location $(\mathrm{p}=$ 0.006 for falx/parasagittal/convexity tumors compared to skull base tumors).

To identify meaningful size cutoff points for risk of higher-grade tumors, we performed RPA. We identified $3.2 \mathrm{~cm}$ as the optimal split, and divided the tumors into groups of $<3 \mathrm{~cm}, 3-6 \mathrm{~cm}$, and $>6 \mathrm{~cm}$. We then performed multivariate logistic regression and calculated odds ratios for the groups (Table 4). Last, we used a multivariate RPA to identify subgroups at highest risk for having a WHO grade II tumor. The k-fold cross-validation was used to determine that 3 splits was the optimal number of splits.

TABLE 3. Univariate logistic regression for predictors of WHO grade II

\begin{tabular}{llcc}
\hline \multicolumn{1}{c}{ Variable } & $\mathrm{OR}^{*}$ & $95 \% \mathrm{Cl}^{*}$ & $\mathrm{p} \mathrm{Value}$ \\
\hline Age & & & 0.61 \\
\hline Tumor size & & & $<0.001 \dagger$ \\
\hline Tumor location & & & \\
\hline$\quad$ Skull base & 1 & & \\
\hline Falx/parasagittal/convexity & 1.90 & $1.38-2.63$ & $<0.001 \dagger$ \\
\hline Other & 1.71 & $1.02-2.86$ & $0.03 \ddagger$ \\
\hline Sex & & & \\
\hline Female & 1 & & \\
\hline Male & 1.94 & $1.41-2.69$ & $<0.001 \ddagger$ \\
\hline
\end{tabular}

* Not calculated for continuous variables.

$\dagger p<0.01$.

$\ddagger p<0.05$.

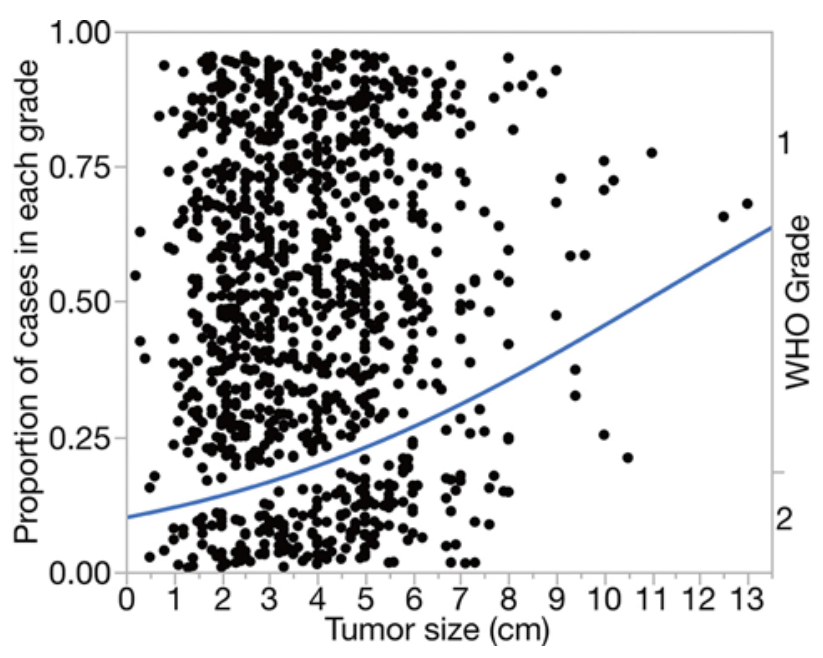

FIG. 1. Scatter plot and logistic regression of tumor grade by tumor size. Each circle represents an individual case and is plotted by tumor size. Tumors above the blue line are WHO grade I, whereas those below are grade II. The blue line is the logistic fit showing that as size increases, the proportion of WHO grade II tumors increases as well $(p<0.001)$.

The first split was tumor size at $3.2 \mathrm{~cm}$, which increased the likelihood of a WHO grade II tumor (23.9\% vs $11.3 \%)$. The second split was on sex, with male patients having an increased likelihood of a WHO grade II tumor compared to female patients $(32.5 \%$ vs $20.0 \%)$. Finally, the third split was made for tumors $<3.2 \mathrm{~cm}$ based on location, with non-skull base tumors having an increased likelihood of a WHO grade II tumor (16.6\% vs 8.8\%) (Fig. 2).

\section{Discussion}

\section{Key Results}

Our results show that larger tumor size is associated with an increased likelihood of a meningioma being WHO grade II, even after controlling for interactions with the known risk factors of sex and meningioma location.

TABLE 4. Multivariate logistic regression for predictors of WHO grade II

\begin{tabular}{|c|c|c|c|}
\hline Variable & OR & $95 \% \mathrm{Cl}$ & p Value \\
\hline \multicolumn{4}{|l|}{ Tumor size } \\
\hline$<3.0 \mathrm{~cm}$ & 1 & & \\
\hline $3.0-6.0 \mathrm{~cm}$ & 1.69 & $1.08-2.66$ & $0.02^{*}$ \\
\hline$>6.0 \mathrm{~cm}$ & 3.01 & $1.53-5.94$ & $0.002 \uparrow$ \\
\hline \multicolumn{4}{|l|}{ Tumor location } \\
\hline Skull base & 1 & & \\
\hline Falx/parasagittal/convexity & 1.83 & $1.19-2.82$ & $0.006 \dagger$ \\
\hline Other & 2.37 & $1.21-4.64$ & $0.02^{*}$ \\
\hline \multicolumn{4}{|l|}{ Sex } \\
\hline Female & 1 & & \\
\hline Male & 1.74 & $1.25-2.43$ & $<0.001 \dagger$ \\
\hline
\end{tabular}




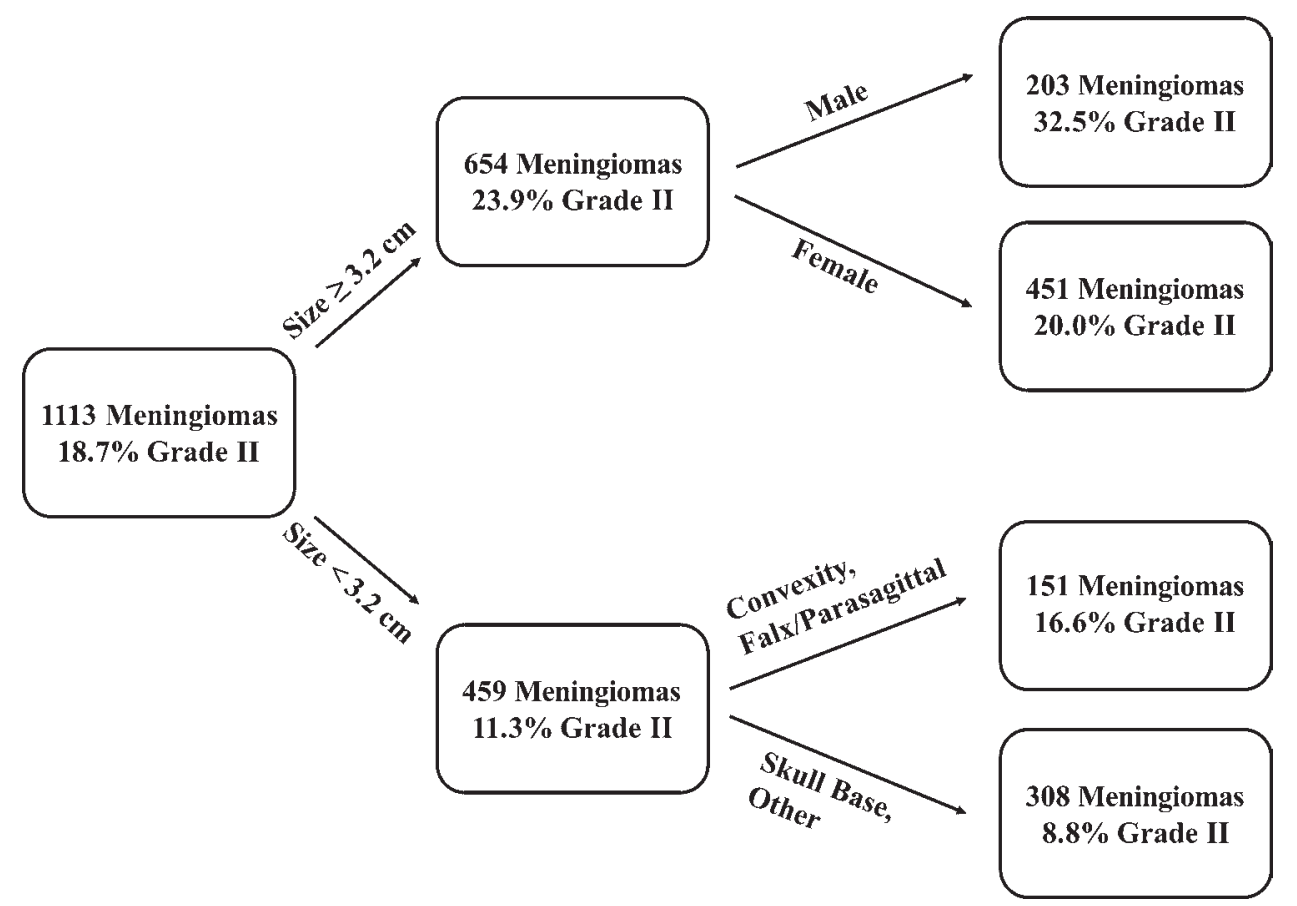

FIG. 2. The RPA for WHO Grade II meningiomas. The optimal number of splits (3) was based on $R^{2}$ calculated by k-fold crossvalidation.

\section{Interpretation of Results}

Here, we show that larger meningiomas are more likely to be WHO grade II tumors. Importantly, this relationship was maintained as an independent risk factor even after crossing with tumor location and male sex in a multivariate analysis. Consistent with prior studies, skull base tumors were less likely to be higher grade than convexity and falx/parasagittal meningiomas. ${ }^{8,19}$ Male sex, another known risk factor for atypical meningioma, was also found to be significant in our study. ${ }^{6,16,19,24}$ As with prior studies, age was not related to WHO grade. Interestingly, the RPA found that among size, sex, and location, tumor size was the most important dividing factor between the likelihood of WHO grade I and II tumors. It also identified a subset of male patients with large tumors who are at particularly high risk. Figure 1 shows how this risk continues to increase with increasing tumor size. These findings are useful when discussing management plans with patients and when preparing expectations for care that may be more likely to include adjuvant radiotherapy. ${ }^{7}$

A recent report found that $20 \%$ of giant meningiomas (i.e., $>5 \mathrm{~cm}$ ) were WHO grade II or III. ${ }^{21}$ Furthermore, in that study, tumor location also influenced recurrence-free survival. Another retrospective analysis reported that tumor size was related to disease-free survival for patients with atypical meningiomas who received radiotherapy. ${ }^{11}$ Our findings add to these prior reports by being the first study to demonstrate that tumor size is directly related to tumor grade.

The findings in this study raise the question of what mechanisms drive the relationship between tumor size and grade. Simplistically thinking, one possibility is that grade II tumors grow more quickly than grade I tumors and are more likely to reach a larger size. However, if that were the case, one might imagine that a faster-growing tumor would present earlier, because rapidly growing tumors often become symptomatic at a smaller size. Alternatively, it is possible that once a slow-growing tumor reaches a large size, a microenvironment develops (perhaps caused by hypoxia) that facilitates the transformation of the tumor to a more aggressive phenotype. Moving forward, we will need to turn to the laboratory to identify what mechanisms drive meningioma growth and cause them to become higher grade.

Significant progress has been made in the last several years to identify what genes are mutated in meningioma. In addition to $N F 2$, mutations have been found in TRAF7, SMO, KLF4, PI3K, and AKT1.1.8 Mutations in POLR2A were identified in recurrent meningiomas. ${ }^{9}$ Multiple independent groups have shown that TERT promoter mutations are associated with shorter time to recurrence, survival, and overall poor prognosis. ${ }^{2,12,13,25}$ In addition to somatic mutations, specific DNA methylation patterns have recently been discovered that complement known chromosomal rearrangements that are associated with tumor grade and survival. ${ }^{4,26}$ In contrast to mechanisms occurring within the meningioma, another possibility is that the immune environment and tumor-immune system interaction may alter tumor growth and drive the development of large size. Interestingly, we recently found that increased PD-L1 expression in meningioma was associated with shorter survival. ${ }^{14}$ We hypothesize that the relationship between meningioma size and grade reflects changes in the molecular landscape of each individual tumor and the way in which individual meningioma interacts with the surrounding microenvironment and immune system. Contin- 
ued basic and translational research efforts are needed to further characterize these drivers of meningioma growth and, we hope, to identify additional molecular targets for treatment.

\section{Limitations of the Study}

This study is limited by its retrospective design. In addition, the criteria for WHO classification changed during the years of this study, and regrading all the samples in this series is not practical. With the 2007 WHO classification, there are more meningiomas classified as grade II, which means that our study probably underestimates the incidence of grade II tumors. ${ }^{27}$ However, the rate of atypical meningiomas in our cohort is similar to that in previously published series. ${ }^{22}$ Another limitation is the use of tumor diameter as a proxy for overall tumor size because meningiomas can take many shapes. Retrospectively performing volumetric analysis was beyond the scope of the current study. Future prospective studies with volumetric analysis and contemporary grading criteria would be an excellent addition to strengthen these results.

\section{Generalizability of the Findings}

One of the most significant strengths of this study is its very large sample size, which makes these results widely generalizable and applicable to all providers treating this disease and to patients who are diagnosed with meningioma.

\section{Conclusions}

In this study, we identified tumor size as an independent risk factor for a meningioma to be WHO grade II. In addition, we recapitulate other studies showing that male sex and tumor location are also risk factors for a meningioma to be WHO grade II. Predicting tumor grade from radiological factors such as preoperative tumor size and location can impact surgical decision making, operative planning, and treatment strategies.

\section{Acknowledgments}

This work was supported by grants from the Linda Wolfe Meningioma Research Fund and the National Cancer Institute of the National Institutes of Health (1F32CA213944-01) to Dr. Magill.

\section{References}

1. Abedalthagafi M, Bi WL, Aizer AA, Merrill PH, Brewster R, Agarwalla PK, et al: Oncogenic PI3K mutations are as common as AKT1 and SMO mutations in meningioma. Neuro Oncol 18:649-655, 2016

2. Abedalthagafi MS, Bi WL, Merrill PH, Gibson WJ, Rose MF, Du Z, et al: ARID1A and TERT promoter mutations in dedifferentiated meningioma. Cancer Genet 208:345-350, 2015

3. Baumgartner JE, Sorenson JM: Meningioma in the pediatric population. J Neurooncol 29:223-228, 1996

4. Bi WL, Zhang M, Wu WW, Mei Y, Dunn IF: Meningioma genomics: diagnostic, prognostic, and therapeutic applications. Front Surg 3:40, 2016

5. Bondy M, Ligon BL: Epidemiology and etiology of intracranial meningiomas: a review. J Neurooncol 29:197-205, 1996
6. Boskos C, Feuvret L, Noel G, Habrand JL, Pommier P, Alapetite $\mathrm{C}$, et al: Combined proton and photon conformal radiotherapy for intracranial atypical and malignant meningioma. Int J Radiat Oncol Biol Phys 75:399-406, 2009

7. Chen WC, Magill ST, Wu A, Vasudevan H, Morin O, Aghi MK, et al: Histopathologic features predict local control of atypical meningioma after surgery and adjuvant radiotherapy. J Neurosurg [in press], 2018

8. Clark VE, Erson-Omay EZ, Serin A, Yin J, Cotney J, Ozduman K, et al: Genomic analysis of non-NF2 meningiomas reveals mutations in TRAF7, KLF4, AKT1, and SMO. Science 339:1077-1080, 2013

9. Clark VE, Harmancı AS, Bai H, Youngblood MW, Lee TI, Baranoski JF, et al: Recurrent somatic mutations in POLR2A define a distinct subset of meningiomas. Nat Genet 48:12531259,2016

10. Claus EB, Bondy ML, Schildkraut JM, Wiemels JL, Wrensch M, Black PM: Epidemiology of intracranial meningioma. Neurosurgery 57:1088-1095, 2005

11. Detti B, Scoccianti S, Di Cataldo V, Monteleone E, Cipressi S, Bordi L, et al: Atypical and malignant meningioma: outcome and prognostic factors in 68 irradiated patients. J Neurooncol 115:421-427, 2013

12. Fürtjes G, Köchling M, Peetz-Dienhart S, Wagner A, Heß $\mathrm{K}$, Hasselblatt M, et al: hTERT promoter methylation in meningiomas and central nervous hemangiopericytomas. J Neurooncol 130:79-87, 2016

13. Goutagny S, Nault JC, Mallet M, Henin D, Rossi JZ, Kalamarides M: High incidence of activating TERT promoter mutations in meningiomas undergoing malignant progression. Brain Pathol 24:184-189, 2014

14. Han SJ, Reis G, Kohanbash G, Shrivastav S, Magill ST, Molinaro AM, et al: Expression and prognostic impact of immune modulatory molecule PD-L1 in meningioma. J Neurooncol 130:543-552, 2016

15. Hasseleid BF, Meling TR, Rønning P, Scheie D, Helseth E: Surgery for convexity meningioma: Simpson Grade I resection as the goal: clinical article. J Neurosurg 117:999-1006, 2012

16. Kano H, Takahashi JA, Katsuki T, Araki N, Oya N, Hiraoka $\mathrm{M}$, et al: Stereotactic radiosurgery for atypical and anaplastic meningiomas. J Neurooncol 84:41-47, 2007

17. Kleihues P, Louis DN, Scheithauer BW, Rorke LB, Reifenberger G, Burger PC, et al: The WHO classification of tumors of the nervous system. J Neuropathol Exp Neurol 61:215-25-229, 2002

18. Kshettry VR, Ostrom QT, Kruchko C, Al-Mefty O, Barnett GH, Barnholtz-Sloan JS: Descriptive epidemiology of World Health Organization grades II and III intracranial meningiomas in the United States. Neuro Oncol 17:1166-1173, 2015

19. Liang RF, Xiu YJ, Wang X, Li M, Yang Y, Mao Q, et al: The potential risk factors for atypical and anaplastic meningiomas: clinical series of 1,239 cases. Int J Clin Exp Med 7:5696-5700, 2014

20. Louis DN, Ohgaki H, Wiestler OD, Cavenee WK, Burger PC, Jouvet A, et al: The 2007 WHO classification of tumours of the central nervous system. Acta Neuropathol 114:97-109, 2007

21. Narayan V, Bir SC, Mohammed N, Savardekar AR, Patra DP, Nanda A: Surgical management of giant intracranial meningioma: operative nuances, challenges and outcome. World Neurosurg 110:e32-e41, 2018

22. Nowak A, Dziedzic T, Krych P, Czernicki T, Kunert P, Marchel A: Benign versus atypical meningiomas: risk factors predicting recurrence. Neurol Neurochir Pol 49:1-10, 2015

23. Rogers L, Barani I, Chamberlain M, Kaley TJ, McDermott M, Raizer J, et al: Meningiomas: knowledge base, treatment outcomes, and uncertainties. A RANO review. J Neurosurg 122:4-23, 2015 
24. Rohringer M, Sutherland GR, Louw DF, Sima AAF: Incidence and clinicopathological features of meningioma. J Neurosurg 71:665-672, 1989

25. Sahm F, Schrimpf D, Olar A, Koelsche C, Reuss D, Bissel $\mathrm{J}$, et al: TERT promoter mutations and risk of recurrence in meningioma. J Natl Cancer Inst 108:djv377, 2015

26. Sahm F, Schrimpf D, Stichel D, Jones DTW, Hielscher T, Schefzyk S, et al: DNA methylation-based classification and grading system for meningioma: a multicentre, retrospective analysis. Lancet Oncol 18:682-694, 2017

27. Smith SJ, Boddu S, Macarthur DC: Atypical meningiomas: WHO moved the goalposts? Br J Neurosurg 21:588-592, 2007

28. von Elm E, Altman DG, Egger M, Pocock SJ, Gøtzsche PC, Vandenbroucke JP: The Strengthening the Reporting of Observational Studies in Epidemiology (STROBE) statement: guidelines for reporting observational studies. PLoS Med 4:e296, 2007

\section{Disclosures}

The authors report no conflict of interest concerning the materi- als or methods used in this study or the findings specified in this paper.

\section{Author Contributions}

Conception and design: Magill, Aghi, Theodosopoulos, McDermott. Acquisition of data: Magill, Young, Chae, Aghi, Theodosopoulos. Analysis and interpretation of data: Magill, Young, Chae, McDermott. Drafting the article: Magill, Young, Chae. Critically revising the article: Magill, Young, McDermott. Reviewed submitted version of manuscript: Magill, Young, McDermott. Approved the final version of the manuscript on behalf of all authors: Magill. Statistical analysis: Magill, Young. Study supervision: Magill, McDermott.

\section{Supplemental Information}

Previous Presentations

A preliminary version of this work was presented at the 2017 North American Skull Base Society Annual Meeting in New Orleans, LA.

\section{Correspondence}

Stephen T. Magill: University of California, San Francisco, CA. stephen.magill@ucsf.edu. 